\title{
EFFECT OF CUTTING HEIGHTS AND MINERAL NPK AND/OR BIOFERTILIZATION TREATMENTS ON MORINGA OLEIFERA, L. PLANTS
}

\author{
A.F. Ali ${ }^{*}$, M.A.H. Abdou ${ }^{* *}$ and O. Rashed ${ }^{*}$ \\ * Department of Horticulture, Fac. Agric., Al-Azhar Univ. (Assuit branch), Egypt. \\ ** Department of Horticulture, Fac. Agric., Minia Univ, Egypt.
}

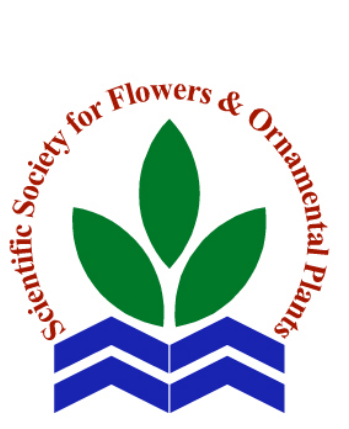

Scientific J. Flowers \& Ornamental Plants, 4(2):209-223 (2017).

Received: 14/5/2017 Accepted: 21/5/2017

ABSTRACT: This study was conducted in Hort. Dept., Fac. Agric., Minia Univ. in the two successive seasons of 2015 and 2016 on Moringa to study the effect of cutting heights $(10,20,30$ and $40 \mathrm{~cm})$ and fertilization on yield productivity and chemical constituents of the plants.

Data indicated that cut plant at $10 \mathrm{~cm}$ was superior than other treatments on increasing branch numbers, fresh and dry weights/plant/cut and total yield/plant/season, as well as, total carbohydrates, protein and vit. C \%. All used six fertilization treatments significantly increased all vegetative growth parameters and chemical constituents as compared with control. The best treatments were NPK (100 \%) and bio. + $75 \%$ NPK.

It could be recommended to cut Moringa oleifera at $10 \mathrm{~cm}$ above soil surface and fertilizing plants with bio. $+75 \%$ NPK dose to obtain a good production biomass and high quality.

Key words: Moringa oleifera, cutting, fertilization, NPK, biofertilization.

\section{INTRODUCTION}

Moringa oleifera, L. (Family Moringaceae) is the most widely cultivated species of the genus Moringa, it is fast growing, considered one of the world's most useful trees as every part of the tree can be used either for food or has other beneficial properties and as a fodder for livestock (Morton, 1991; Anwar et al., 2007 and Nouman et al., 2012).

Few studies about the height this plant should be harvested to get good yields and biomass quality are available. Moringa has high speed and regrowth capacity, further cuts are suggested to be 10 to $40 \mathrm{~cm}$ above soil surface to obtain a high yield in production of fresh biomass (Reyes, 2006; Foidl et al., 2011; Santiesteban et al., 2012 and Padilla et al., 2014).

Biofertilizers are considered to be low cost, eco-friendly are renewable sources of plant nutrients supplementing chemical fertilizers in sustainable agricultural system (Hedge et al., 1999). A good number of researchers concluded that biofertilizers enhanced the growth and quality of mass production of Moringa (Dash and Gupta, 2009; Asaolu et al., 2012; Zayed, 2012; Darwish, 2015 and Mosaad, 2016).

Chemical nutrients especially N, P and K are very important for plants, because NPK increased plant growth and chemical constituents as emphasized by Dash and Gupta (2009); Fagbenro et al. (2013); Abdullahi et al. (2013); Umar (2014); Darwish (2015) and Mosaad (2016) on Moringa.

The objective of this research was to determine the best height of cutting and Moringa oleifera fertilization. 


\section{A.F. Ali et al.}

\section{MATERIALS AND METHODS}

The experiment was carried out during the two successive seasons of 2015 and 2016 at the Floriculture Nursery at the Experimental Farm and in the Laboratory of Floriculture and Food Industries Department, Fac. of Agric., Minia Univ. to investigate the effect of cut height and bio. and/or mineral NPK fertilization treatments, as well as, their interaction on growth and chemical constituents of Moringa oleifera grown in sandy calcareous soil. The seeds of Moringa oleifera Lam. were obtained from Nursery of Ornamental Plants Division, Horticulture Department, Faculty of Agriculture, Minia University. Physical and chemical properties of the used soil in both seasons were determined according to Jackson (1973) and are shown in Table (a).

The experiment was arranged in a randomized complete block design in a splitplot design with three replicates each replicate containing 3 seedlings. The main plots (A) included four levels of cutting heights, while, seven biofertilizers and/or mineral NPK fertilization treatments (B) occupied the sub-plots, therefore, the interaction treatments $(\mathrm{A} \times \mathrm{B})$ were 28 treatments.

\section{Treatments:}

The main-plots (A) were as follows: Cutting heights at 10, 20, 30 and $40 \mathrm{~cm}$ above soil surface.
The sub-plots (B) were as follows: Control (without fertilizer), $100 \%$ NPK as recommended dose (Abdou et al., 2016), 75\% NPK, 50\% NPK, biofertilizers (Minia Azotein + phosphorein), biofertilizers $+75 \%$ NPK and biofertilizers $+50 \%$ NPK.

\section{Data recorded:}

Number of branches per plant, fresh and dry weights of aerial parts/cut and yield of Moringa fresh and dry weights/season, as well as, total carbohydrates \% according to (Michel et al., 1956), protein \% (Wilde et al., 1985 to determined $\mathrm{N} \%$, then $\mathrm{N} \%$ was multiplied by 6.25 to obtain protein \%) and vit. C (Roe and Keuther, 1953).

Seeds were sown in the first day of March for both seasons in containers $(25 \times 25 \times 35 \mathrm{~cm})$ each filled with $20 \mathrm{~kg}$ of sandy calcareous soil plus $20 \mathrm{~g}$ of compost in the Nursery of Ornamental Plants, each container contain three seeds and seedlings were thinned to one seedling/container after four weeks from sowing date ( $28^{\text {th }}$ March).

Fresh and active biofertilizers, Minia Azotein (M.A., containing $\mathrm{N}$-fixing bacteria) and phosphorein (containing phosphate dissolving bacteria) were obtained from the Laboratory of Biofertilizers, Department of Genetic, Fac. of Agric., Minia University. Biofertilizers were applied three times to the soil beside the plants at the rate of 50 $\mathrm{cm}^{3} /$ hill ( $1 \mathrm{ml}=10^{7}$ cells of bacteria).

Table a. Physical and chemical properties of the used soil.

\begin{tabular}{|c|c|c|c|c|c|c|}
\hline \multirow{2}{*}{ Character } & \multicolumn{2}{|c|}{ Value } & \multirow{2}{*}{\multicolumn{2}{|c|}{ Character }} & \multicolumn{2}{|c|}{ Value } \\
\hline & 2015 & 2016 & & & 2015 & 2016 \\
\hline Sand \% & 88.00 & 89.00 & Total N \% & & 0.02 & 0.02 \\
\hline Silt \% & 8.30 & 7.40 & Available 1 & & 3.25 & 3.56 \\
\hline Clay \% & 3.70 & 3.60 & Extr. K (n & soil) & 0.90 & 1.01 \\
\hline Texture & Sandy & Sandy & & & & \\
\hline $\mathrm{CaCO}_{3} \%$ & 14.42 & 14.91 & & $\mathbf{F e}$ & 1.10 & 1.18 \\
\hline pH (1 : 2.5) & 8.17 & 8.21 & DTPA & $\mathbf{C u}$ & 0.39 & 0.43 \\
\hline Organic matter \% & 0.06 & 0.05 & Ext. ppm & Zn & 0.36 & 0.30 \\
\hline E.C. (m mhos/cm) & 1.09 & 1.11 & & Mn & 0.60 & 0.71 \\
\hline
\end{tabular}


The first dose, for both M.A. and phos. was added 32 days from sowing date ( $2^{\text {nd }}$ April) and repeated 60 days thereafter $\left(2^{\text {nd }}\right.$ June and $2^{\text {nd }}$ August) and then plants were irrigated immediately. All other agricultural practices were performed as usual.

Mineral NPK was used as $6 \mathrm{~g} /$ container of ammonium sulphate $(20.50 \% \mathrm{~N})$ (equal $300 \mathrm{~kg} / \mathrm{fed}), \quad 3 \mathrm{~g} /$ container calcium superphosphate $\left(15.5 \% \mathrm{P}_{2} \mathrm{O}_{5}\right)$ (equal 150 $\mathrm{kg} / \mathrm{fed}$ ) and $1.5 \mathrm{~g} /$ container potassium sulphate $\left(48 \% \mathrm{~K}_{2} \mathrm{O}\right)$ (equal $75 \mathrm{~kg} /$ fed) for the treatment of $100 \%$ mineral NPK. For $75 \%$ mineral NPK was represented by 4.5, 2.25 and $1.125 \mathrm{~g} /$ container. Also, 50\% mineral NPK was 3, 1.5 and $0.75 \mathrm{~g} /$ container of the above mentioned three fertilizers.

The amounts of NK were divided to three equal batches and added after 7 days from each biofertilizers treatment (9 April, 9 June and 9 August). All amounts of P were added during preparing the soil to sow the seeds for each season.

During each experimental season the plants were harvested three times. In each harvest, the plants were cut leaving about 10 , 20, 30 and $40 \mathrm{~cm}$ above the soil surface according to the treatments occupied in the main plots. The first cut was done on $1^{\text {st }}$ of June. Meanwhile, the second cut was done in $1^{\text {st }}$ of August and the third cut was done October $1^{\text {st }}$ in the two growing seasons.

The obtained data were tabulated and statistically analyzed according to MSTATC (1986) and the L.S.D. test at $5 \%$ was followed to compare between the means.

\section{RESULTS AND DISCUSSION}

\section{1- Vegetative growth parameters:}

Data presented in Tables (1 to 8) indicated that number of branches/cut, fresh and dry weights/cut and yield of aerial parts of Moringa oleifera/season) were statistically influenced by cutting height either during the three cuts or during the first and second seasons. The highest values were obtained when plants were cut at $10 \mathrm{~cm}$ high.
The increase in yield production with decreasing cutting height can possibly be attributed to the fact that plants harvested at $10 \mathrm{~cm}$ high contain much more branches. Hence, the $10 \mathrm{~cm}$ cut was the higher quality as reflected in the higher production. Similar results were obtained by Reyes (2006); Padilla et al. (2014) and Lawal et al. (2015) on Moringa oleifera.

Concerning the effect mineral NPK and/or biofertilization treatments, data in Tables (1 to 6) indicated that all used six treatments significantly increased number of branches/cut, aerial parts fresh and dry weights/plant comparing with control during the three cuts, consequently the total yield of Moringa either fresh or dry weights per season as shown in Tables (7 and 8). The highest values were obtained with mineral NPK (full dose) or bio. + 75\% NPK in both seasons. Similar results were obtained by Dash and Gupta (2009); Fagbenro et al. (2013) and Mosaad (2016) on Moringa, Kohan et al. (2000) on poplar and Singh (2015) on Leucaena leucocephala, concerning the effect of mineral NPK. Biofertilization treatments were effective on increasing number of branches and fresh and dry weights production as reported by Dash and Gupta (2009), Zayed (2012) and Darwish (2015) on Moringa, Moustafa (2008) on Chorisia speciosa, Abdou and Ashour (2012) on jojoba and Soliman et al. (2015) on Delonix regia.

The interaction between main and sub plots $(A \times B)$ was significant for number of branches, fresh and dry weights/cut and yield of Moringa oleifera per season (Tables, 1 to $8)$. The best interaction treatments were obtained when Moringa was cut at $10 \mathrm{~cm}$ above soil surface and fertilized with bio. + $75 \%$ NPK dose or mineral NPK at full dose.

\section{2- Chemical constituents:}

Data presented in Tables (9 and 10) indicated that cutting heights had no significant effects on total carbohydrates and protein \% in Moringa leaves. For vit. C 
Table 1. Effect of cutting heights and some bio. and/or mineral NPK fertilization treatments on number of branches/plant of moringa during 2015 season.

\begin{tabular}{|c|c|c|c|c|c|}
\hline \multicolumn{6}{|c|}{$\mathbf{1}^{\text {st }}$ Cut } \\
\hline \multirow{2}{*}{$\begin{array}{l}\text { Some bio. and/or mineral } \\
\text { fertilization treatments (B) }\end{array}$} & \multicolumn{5}{|c|}{ Cutting heights (cm) (A) } \\
\hline & 10 & 20 & 30 & 40 & Mean (B) \\
\hline Control & 6.0 & 4.3 & 2.3 & 2.0 & 3.7 \\
\hline NPK 100\% & 14.3 & 12.3 & 10.0 & 8.7 & 11.3 \\
\hline NPK 75\% & 12.3 & 9.7 & 8.0 & 6.0 & 9.0 \\
\hline NPK 50\% & 9.0 & 7.0 & 4.7 & 4.0 & 6.2 \\
\hline Bio. & 7.7 & 5.7 & 3.7 & 3.0 & 5.0 \\
\hline Bio. + NPK 75\% & 13.3 & 11.0 & 9.0 & 7.7 & 10.3 \\
\hline Bio. + NPK 50\% & 10.0 & 8.3 & 6.0 & 5.0 & 7.3 \\
\hline Mean (A) & 10.4 & 8.3 & 6.2 & 5.2 & 7.5 \\
\hline L.S.D. at $5 \%$ & \multicolumn{2}{|c|}{ A : 2.1} & B : 0.5 & \multicolumn{2}{|r|}{$\mathrm{AB}: 1.0$} \\
\hline \multicolumn{6}{|c|}{$2^{\text {nd }}$ Cut } \\
\hline Control & 8.0 & 7.0 & 4.7 & 2.3 & 5.5 \\
\hline NPK $100 \%$ & 16.0 & 14.3 & 13.0 & 10.7 & 13.5 \\
\hline NPK 75\% & 13.7 & 12.3 & 10.0 & 7.7 & 10.9 \\
\hline NPK 50\% & 11.0 & 9.7 & 8.0 & 5.3 & 8.5 \\
\hline Bio. & 9.7 & 8.7 & 6.7 & 4.3 & 7.3 \\
\hline Bio. + NPK 75\% & 15.0 & 13.3 & 11.3 & 8.7 & 12.1 \\
\hline Bio. + NPK 50\% & 12.0 & 11.3 & 9.0 & 6.7 & 9.8 \\
\hline Mean (A) & 12.2 & 11.0 & 9.0 & 6.5 & 9.7 \\
\hline L.S.D. at $5 \%$ & \multicolumn{2}{|c|}{ A : 1.5} & B : 0.5 & \multicolumn{2}{|r|}{$\mathrm{AB}: 1.0$} \\
\hline \multicolumn{6}{|c|}{$3^{\text {rd }}$ Cut } \\
\hline Control & 10.0 & 9.0 & 7.0 & 4.3 & 7.6 \\
\hline NPK 100\% & 18.0 & 17.3 & 15.0 & 11.0 & 15.3 \\
\hline NPK 75\% & 16.0 & 15.0 & 12.7 & 9.0 & 13.2 \\
\hline NPK 50\% & 13.0 & 11.7 & 10.0 & 7.0 & 10.4 \\
\hline Bio. & 12.0 & 10.7 & 9.0 & 6.0 & 9.4 \\
\hline Bio. + NPK 75\% & 17.0 & 16.0 & 13.7 & 10.0 & 14.2 \\
\hline Bio. + NPK 50\% & 14.0 & 13.0 & 11.3 & 8.0 & 11.6 \\
\hline Mean (A) & 14.3 & 13.2 & 11.2 & 7.9 & 11.7 \\
\hline L.S.D. at $5 \%$ & \multicolumn{2}{|c|}{ A : 0.8} & B : 0.3 & \multicolumn{2}{|r|}{$\mathrm{AB}: 0.6$} \\
\hline
\end{tabular}

Bio. : Biofertilizers (Minia Azotein + phosphorein) 
Table 2. Effect of cutting heights and some bio. and/or mineral NPK fertilization treatments on number of branches/plant of moringa during 2016 season.

\begin{tabular}{|c|c|c|c|c|c|}
\hline \multicolumn{6}{|c|}{$1^{\text {st }}$ Cut } \\
\hline \multirow{2}{*}{$\begin{array}{l}\text { Some bio. and/or mineral } \\
\text { fertilization treatments (B) }\end{array}$} & \multicolumn{5}{|c|}{ Cutting heights (cm) (A) } \\
\hline & 10 & 20 & 30 & 40 & Mean (B) \\
\hline Control & 5.7 & 4.0 & 2.3 & 1.7 & 3.4 \\
\hline NPK $100 \%$ & 14.7 & 13.7 & 13.0 & 9.7 & 12.8 \\
\hline NPK 75\% & 12.0 & 10.0 & 7.7 & 7.0 & 9.2 \\
\hline NPK 50\% & 9.0 & 7.0 & 5.0 & 4.3 & 6.3 \\
\hline Bio. & 7.7 & 6.0 & 3.7 & 3.0 & 5.1 \\
\hline Bio. + NPK 75\% & 13.3 & 11.0 & 9.7 & 8.0 & 10.5 \\
\hline Bio. + NPK 50\% & 10.0 & 8.7 & 6.3 & 6.0 & 7.8 \\
\hline Mean (A) & 10.3 & 8.6 & 6.8 & 5.7 & 7.9 \\
\hline L.S.D. at $5 \%$ & \multicolumn{2}{|c|}{ A : 1.5} & B : 0.8 & \multicolumn{2}{|r|}{$\mathrm{AB}: 1.6$} \\
\hline \multicolumn{6}{|c|}{$2^{\text {nd }}$ Cut } \\
\hline Control & 8.7 & 8.0 & 6.7 & 5.0 & 7.1 \\
\hline NPK 100\% & 18.0 & 16.7 & 14.7 & 12.0 & 15.3 \\
\hline NPK 75\% & 15.3 & 14.0 & 11.7 & 9.7 & 12.7 \\
\hline NPK $50 \%$ & 12.3 & 11.3 & 9.7 & 7.3 & 10.2 \\
\hline Bio. & 11.0 & 10.3 & 8.3 & 6.0 & 8.9 \\
\hline Bio. + NPK 75\% & 16.7 & 15.7 & 13.0 & 10.7 & 14.0 \\
\hline Bio. + NPK 50\% & 14.0 & 13.0 & 10.7 & 8.7 & 11.6 \\
\hline Mean (A) & 13.7 & 12.7 & 10.7 & 8.5 & 11.4 \\
\hline L.S.D. at 5\% & \multicolumn{2}{|c|}{ A : 0.9} & B : 0.8 & \multicolumn{2}{|r|}{$\mathrm{AB}: 1.6$} \\
\hline \multicolumn{6}{|c|}{$3^{\text {rd }}$ Cut } \\
\hline Control & 10.7 & 9.7 & 7.3 & 5.0 & 8.2 \\
\hline NPK 100\% & 19.0 & 17.7 & 15.3 & 12.0 & 16.0 \\
\hline NPK 75\% & 16.7 & 15.0 & 13.3 & 10.0 & 13.8 \\
\hline NPK 50\% & 13.3 & 12.3 & 11.0 & 7.3 & 11.0 \\
\hline Bio. & 12.3 & 11.0 & 10.0 & 6.3 & 9.9 \\
\hline Bio. + NPK 75\% & 18.0 & 16.0 & 14.3 & 11.0 & 14.8 \\
\hline Bio. + NPK 50\% & 14.7 & 13.7 & 12.0 & 9.0 & 12.3 \\
\hline Mean (A) & 15.0 & 13.6 & 11.9 & 8.7 & 12.3 \\
\hline L.S.D. at 5\% & \multicolumn{2}{|c|}{ A : 1.1} & B : 0.4 & \multicolumn{2}{|r|}{$\mathrm{AB}: 0.8$} \\
\hline
\end{tabular}

Bio. : Biofertilizers (Minia Azotein + phosphorein) 
Table 3. Effect of cutting heights and some bio. and/or mineral NPK fertilization treatments on fresh weight $(\mathrm{kg})$ of moringa during 2015 season.

\begin{tabular}{|c|c|c|c|c|c|}
\hline \multicolumn{6}{|c|}{$1^{\text {st }}$ Cut } \\
\hline \multirow{2}{*}{$\begin{array}{l}\text { Some bio. and/or mineral } \\
\text { fertilization treatments (B) }\end{array}$} & \multicolumn{5}{|c|}{ Cutting heights (cm) (A) } \\
\hline & 10 & 20 & 30 & 40 & Mean (B) \\
\hline Control & 4.25 & 1.66 & 1.62 & 0.74 & 2.07 \\
\hline NPK $100 \%$ & 12.92 & 6.14 & 5.66 & 3.14 & 6.97 \\
\hline NPK 75\% & 8.56 & 3.75 & 3.56 & 2.28 & 4.54 \\
\hline NPK 50\% & 4.85 & 1.92 & 1.88 & 1.82 & 2.62 \\
\hline Bio. & 4.54 & 1.83 & 1.80 & 1.60 & 2.44 \\
\hline Bio. + NPK 75\% & 13.16 & 5.56 & 5.38 & 3.61 & 6.93 \\
\hline Bio. + NPK 50\% & 6.68 & 3.10 & 2.91 & 2.19 & 3.72 \\
\hline Mean (A) & 7.85 & 3.42 & 3.26 & 2.20 & 4.18 \\
\hline L.S.D. at $5 \%$ & \multicolumn{2}{|c|}{ A $: 0.10$} & B : 0.12 & \multicolumn{2}{|r|}{$\mathrm{AB}: 0.24$} \\
\hline \multicolumn{6}{|c|}{$2^{\text {nd }}$ Cut } \\
\hline Control & 4.54 & 1.79 & 1.77 & 1.62 & 2.43 \\
\hline NPK $100 \%$ & 11.41 & 7.48 & 7.31 & 4.37 & 7.64 \\
\hline NPK 75\% & 7.68 & 4.64 & 4.63 & 3.38 & 5.08 \\
\hline NPK $50 \%$ & 5.40 & 2.86 & 2.83 & 2.75 & 3.46 \\
\hline Bio. & 5.09 & 2.82 & 2.46 & 2.42 & 3.20 \\
\hline Bio. + NPK 75\% & 12.43 & 6.44 & 6.34 & 4.80 & 7.50 \\
\hline Bio. + NPK 50\% & 5.85 & 4.27 & 3.31 & 3.09 & 4.13 \\
\hline Mean (A) & 7.49 & 4.33 & 4.09 & 3.20 & 4.78 \\
\hline L.S.D. at $5 \%$ & \multicolumn{2}{|c|}{ A $: 0.20$} & B : 0.51 & \multicolumn{2}{|r|}{$\mathrm{AB}: 1.02$} \\
\hline \multicolumn{6}{|c|}{$3^{\text {rd }}$ Cut } \\
\hline Control & 5.54 & 2.69 & 2.46 & 2.03 & 3.18 \\
\hline NPK 100\% & 11.79 & 8.14 & 7.69 & 5.50 & 8.28 \\
\hline NPK 75\% & 8.68 & 5.36 & 5.25 & 4.82 & 6.03 \\
\hline NPK 50\% & 6.64 & 3.73 & 3.66 & 3.30 & 4.33 \\
\hline Bio. & 6.38 & 3.23 & 3.06 & 2.85 & 3.88 \\
\hline Bio. + NPK 75\% & 12.64 & 6.86 & 6.72 & 5.90 & 8.03 \\
\hline Bio. + NPK 50\% & 7.46 & 4.62 & 4.22 & 3.94 & 5.06 \\
\hline Mean (A) & 8.45 & 4.95 & 4.72 & 4.05 & 5.54 \\
\hline L.S.D. at 5\% & \multicolumn{2}{|c|}{ A : 0.67} & B : 0.43 & & $\mathrm{AB}: 0.86$ \\
\hline
\end{tabular}

Bio. : Biofertilizers (Minia Azotein + phosphorein) 
Table 4. Effect of cutting heights and some bio. and/or mineral NPK fertilization treatments on fresh weight $(\mathrm{kg})$ of moringa during 2016 season.

\begin{tabular}{|c|c|c|c|c|c|}
\hline \multicolumn{6}{|c|}{$\mathbf{1}^{\text {st }}$ Cut } \\
\hline \multirow{2}{*}{$\begin{array}{l}\text { Some bio. and/or mineral } \\
\text { fertilization treatments (B) }\end{array}$} & \multicolumn{5}{|c|}{ Cutting heights (cm) (A) } \\
\hline & 10 & 20 & 30 & 40 & Mean (B) \\
\hline Control & 5.29 & 2.74 & 2.10 & 1.84 & 2.99 \\
\hline NPK 100\% & 13.00 & 11.16 & 9.67 & 8.23 & 10.52 \\
\hline NPK 75\% & 9.59 & 7.67 & 7.00 & 4.73 & 7.25 \\
\hline NPK 50\% & 5.92 & 3.17 & 3.07 & 2.98 & 3.79 \\
\hline Bio. & 5.61 & 2.94 & 2.80 & 2.69 & 3.51 \\
\hline Bio. + NPK 75\% & 12.22 & 11.60 & 9.60 & 5.96 & 9.85 \\
\hline Bio. + NPK 50\% & 7.74 & 6.33 & 6.00 & 4.15 & 6.06 \\
\hline Mean (A) & 8.48 & 6.52 & 5.75 & 4.37 & 6.28 \\
\hline L.S.D. at $5 \%$ & \multicolumn{2}{|c|}{ A : 0.72} & B : 0.53 & \multicolumn{2}{|r|}{$\mathrm{AB}: 1.06$} \\
\hline \multicolumn{6}{|c|}{$2^{\text {nd }}$ Cut } \\
\hline Control & 8.67 & 8.00 & 3.78 & 3.59 & 6.01 \\
\hline NPK $100 \%$ & 12.52 & 8.38 & 8.15 & 6.11 & 8.79 \\
\hline NPK 75\% & 8.03 & 5.97 & 5.86 & 5.23 & 6.27 \\
\hline NPK 50\% & 6.31 & 5.22 & 4.53 & 4.52 & 5.15 \\
\hline Bio. & 6.03 & 4.13 & 4.00 & 3.93 & 4.52 \\
\hline Bio. + NPK 75\% & 12.79 & 8.33 & 7.38 & 6.55 & 8.76 \\
\hline Bio. + NPK 50\% & 6.80 & 5.79 & 5.50 & 4.70 & 5.70 \\
\hline Mean (A) & 8.74 & 6.55 & 5.60 & 4.95 & 6.46 \\
\hline L.S.D. at 5\% & \multicolumn{2}{|c|}{ A : 0.38} & B : 0.56 & \multicolumn{2}{|r|}{$\mathrm{AB}: 1.12$} \\
\hline \multicolumn{6}{|c|}{$3^{\text {rd }}$ Cut } \\
\hline Control & 5.61 & 3.97 & 3.91 & 3.81 & 4.33 \\
\hline NPK 100\% & 13.23 & 8.96 & 8.95 & 6.56 & 9.43 \\
\hline NPK 75\% & 8.54 & 6.14 & 5.84 & 5.24 & 6.44 \\
\hline NPK 50\% & 6.75 & 4.84 & 4.55 & 4.53 & 5.17 \\
\hline Bio. & 6.40 & 4.53 & 4.26 & 4.08 & 4.82 \\
\hline Bio. + NPK 75\% & 14.03 & 7.99 & 7.03 & 8.21 & 9.32 \\
\hline Bio. + NPK 50\% & 7.62 & 5.44 & 5.24 & 4.95 & 5.81 \\
\hline Mean (A) & 8.88 & 5.98 & 5.68 & 5.34 & 6.47 \\
\hline L.S.D. at $5 \%$ & \multicolumn{2}{|c|}{ A : 0.52} & B : 0.40 & \multicolumn{2}{|r|}{$\mathrm{AB}: 0.80$} \\
\hline
\end{tabular}

Bio. : Biofertilizers (Minia Azotein + phosphorein) 
Table 5. Effect of cutting heights and some bio. and/or mineral NPK fertilization treatments on dry weight (kg) of moringa during 2015 season.

\begin{tabular}{|c|c|c|c|c|c|}
\hline \multicolumn{6}{|c|}{$1^{\text {st }}$ Cut } \\
\hline \multirow{2}{*}{$\begin{array}{l}\text { Some bio. and/or mineral } \\
\text { fertilization treatments (B) }\end{array}$} & \multicolumn{5}{|c|}{ Cutting heights (cm) (A) } \\
\hline & 10 & 20 & 30 & 40 & Mean (B) \\
\hline Control & 1.56 & 0.61 & 0.60 & 0.27 & 0.76 \\
\hline NPK 100\% & 3.83 & 2.63 & 2.45 & 1.34 & 2.56 \\
\hline NPK 75\% & 3.15 & 1.38 & 1.31 & 0.84 & 1.67 \\
\hline NPK 50\% & 1.78 & 0.71 & 0.69 & 0.67 & 0.96 \\
\hline Bio. & 1.67 & 0.67 & 0.66 & 0.59 & 0.90 \\
\hline Bio. + NPK 75\% & 4.84 & 2.04 & 1.97 & 1.33 & 2.55 \\
\hline Bio. + NPK 50\% & 2.46 & 1.14 & 1.07 & 0.81 & 1.37 \\
\hline Mean (A) & 2.76 & 1.31 & 1.25 & 0.84 & 1.54 \\
\hline L.S.D. at $5 \%$ & \multicolumn{2}{|c|}{ A : 0.26} & B : 0.51 & \multicolumn{2}{|r|}{$\mathrm{AB}: 1.02$} \\
\hline \multicolumn{6}{|c|}{$2^{\text {nd }}$ Cut } \\
\hline Control & 1.67 & 0.66 & 0.65 & 0.60 & 0.90 \\
\hline NPK 100\% & 4.19 & 2.75 & 2.69 & 1.61 & 2.81 \\
\hline NPK 75\% & 2.82 & 1.71 & 1.70 & 1.24 & 1.87 \\
\hline NPK $50 \%$ & 1.99 & 1.05 & 1.04 & 1.01 & 1.27 \\
\hline Bio. & 1.87 & 1.04 & 0.90 & 0.89 & 1.18 \\
\hline Bio. + NPK 75\% & 4.56 & 2.37 & 2.33 & 1.77 & 2.76 \\
\hline Bio. + NPK 50\% & 2.15 & 1.57 & 1.22 & 1.14 & 1.52 \\
\hline Mean (A) & 2.75 & 1.59 & 1.50 & 1.18 & 1.76 \\
\hline L.S.D. at 5\% & \multicolumn{2}{|c|}{ A : 0.10} & B : 0.19 & \multicolumn{2}{|r|}{$\mathrm{AB}: 0.38$} \\
\hline \multicolumn{6}{|c|}{$3^{\text {rd }}$ Cut } \\
\hline Control & 2.04 & 0.99 & 0.90 & 0.75 & 1.17 \\
\hline NPK 100\% & 4.33 & 2.99 & 2.83 & 2.02 & 3.04 \\
\hline NPK 75\% & 3.19 & 1.97 & 1.93 & 1.77 & 2.22 \\
\hline NPK 50\% & 2.44 & 1.37 & 1.35 & 1.21 & 1.59 \\
\hline Bio. & 2.35 & 1.19 & 1.13 & 1.05 & 1.43 \\
\hline Bio. + NPK 75\% & 4.64 & 2.52 & 2.47 & 2.17 & 2.95 \\
\hline Bio. + NPK 50\% & 2.74 & 1.70 & 1.55 & 1.45 & 1.86 \\
\hline Mean (A) & 3.10 & 1.82 & 1.74 & 1.49 & 2.04 \\
\hline L.S.D. at $5 \%$ & \multicolumn{2}{|c|}{ A : 0.12} & B : 0.16 & & $\mathrm{AB}: 0.32$ \\
\hline
\end{tabular}

Bio. : Biofertilizers (Minia Azotein + phosphorein) 
Table 6. Effect of cutting heights and some bio. and/or mineral NPK fertilization treatments on dry weight (kg) of moringa during 2016 season.

\begin{tabular}{|c|c|c|c|c|c|}
\hline \multicolumn{6}{|c|}{$\mathbf{1}^{\text {st }}$ Cut } \\
\hline \multirow{2}{*}{$\begin{array}{l}\text { Some bio. and/or mineral } \\
\text { fertilization treatments (B) }\end{array}$} & \multicolumn{5}{|c|}{ Cutting heights (cm) (A) } \\
\hline & 10 & 20 & 30 & 40 & Mean (B) \\
\hline Control & 1.94 & 1.01 & 0.77 & 0.68 & 1.10 \\
\hline NPK 100\% & 4.78 & 4.10 & 3.56 & 3.03 & 3.87 \\
\hline NPK 75\% & 3.53 & 2.82 & 2.57 & 1.74 & 2.67 \\
\hline NPK 50\% & 2.18 & 1.17 & 1.13 & 1.10 & 1.40 \\
\hline Bio. & 2.06 & 1.08 & 1.03 & 0.99 & 1.29 \\
\hline Bio. + NPK 75\% & 4.49 & 4.27 & 3.53 & 2.20 & 3.62 \\
\hline Bio. + NPK 50\% & 2.85 & 2.33 & 2.21 & 1.53 & 2.23 \\
\hline Mean (A) & 3.12 & 2.40 & 2.11 & 1.61 & 2.31 \\
\hline L.S.D. at $5 \%$ & \multicolumn{2}{|c|}{ A : 0.08} & B : 0.07 & \multicolumn{2}{|r|}{$\mathrm{AB}: 0.14$} \\
\hline \multicolumn{6}{|c|}{$2^{\text {nd }}$ Cut } \\
\hline Control & 3.19 & 2.94 & 1.39 & 1.32 & 2.21 \\
\hline NPK 100\% & 4.60 & 3.08 & 3.00 & 2.25 & 3.23 \\
\hline NPK 75\% & 2.95 & 2.19 & 2.15 & 1.92 & 2.30 \\
\hline NPK $50 \%$ & 2.32 & 1.92 & 1.67 & 1.66 & 1.89 \\
\hline Bio. & 2.22 & 1.52 & 1.47 & 1.44 & 1.66 \\
\hline Bio. + NPK 75\% & 4.70 & 3.06 & 2.71 & 2.41 & 3.22 \\
\hline Bio. + NPK 50\% & 2.50 & 2.13 & 2.02 & 1.73 & 2.10 \\
\hline Mean (A) & 3.21 & 2.41 & 2.06 & 1.82 & 2.37 \\
\hline L.S.D. at 5\% & \multicolumn{2}{|c|}{ A : 0.13} & B : 0.08 & \multicolumn{2}{|r|}{$\mathrm{AB}: 0.16$} \\
\hline \multicolumn{6}{|c|}{$3^{\text {rd }}$ Cut } \\
\hline Control & 2.06 & 1.46 & 1.44 & 1.40 & 1.59 \\
\hline NPK 100\% & 4.86 & 3.29 & 3.29 & 2.41 & 3.46 \\
\hline NPK 75\% & 3.14 & 2.26 & 2.15 & 1.93 & 2.37 \\
\hline NPK 50\% & 2.48 & 1.78 & 1.67 & 1.67 & 1.90 \\
\hline Bio. & 2.35 & 1.67 & 1.57 & 1.50 & 1.77 \\
\hline Bio. + NPK 75\% & 5.16 & 2.94 & 2.58 & 3.01 & 3.42 \\
\hline Bio. + NPK 50\% & 2.80 & 2.00 & 1.93 & 1.82 & 2.14 \\
\hline Mean (A) & 3.26 & 2.20 & 2.09 & 1.96 & 2.38 \\
\hline L.S.D. at $5 \%$ & \multicolumn{2}{|c|}{ A : 0.32} & B : 0.37 & & $\mathrm{AB}: 0.74$ \\
\hline
\end{tabular}

Bio. : Biofertilizers (Minia Azotein + phosphorein) 
Table 7. Effect of cutting heights and some bio. and/or mineral NPK fertilization treatments on total fresh weight (kg/plant/season) of moringa during 2015 and 2016 seasons.

\begin{tabular}{|c|c|c|c|c|c|}
\hline \multicolumn{6}{|c|}{$1^{\text {st }}$ season (2015) } \\
\hline \multirow{2}{*}{$\begin{array}{l}\text { Some bio. and/or mineral } \\
\text { fertilization treatments (B) }\end{array}$} & \multicolumn{5}{|c|}{ Cutting heights (cm) (A) } \\
\hline & 10 & 20 & 30 & 40 & Mean (B) \\
\hline Control & 14.33 & 6.14 & 5.85 & 4.39 & 7.68 \\
\hline NPK $100 \%$ & 36.12 & 21.76 & 20.66 & 13.01 & 22.89 \\
\hline NPK 75\% & 24.92 & 13.75 & 13.44 & 10.48 & 15.65 \\
\hline NPK 50\% & 16.89 & 8.51 & 8.37 & 7.87 & 10.41 \\
\hline Bio. & 16.01 & 7.88 & 7.32 & 6.87 & 9.52 \\
\hline Bio. + NPK 75\% & 38.22 & 18.86 & 18.44 & 14.31 & 22.46 \\
\hline Bio. + NPK 50\% & 19.99 & 11.99 & 10.44 & 9.22 & 12.91 \\
\hline Mean (A) & 23.78 & 12.70 & 12.07 & 9.45 & \\
\hline L.S.D. at $5 \%$ & \multicolumn{2}{|c|}{ A : 0.63} & B : 1.09 & \multicolumn{2}{|r|}{$\mathrm{AB}: 2.18$} \\
\hline \multicolumn{6}{|c|}{$2^{\text {nd }}$ season $(2016)$} \\
\hline Control & 19.57 & 14.71 & 9.79 & 9.24 & 13.33 \\
\hline NPK $100 \%$ & 38.75 & 28.50 & 26.77 & 20.90 & 28.73 \\
\hline NPK 75\% & 26.16 & 19.78 & 18.70 & 15.20 & 19.96 \\
\hline NPK 50\% & 18.98 & 13.23 & 12.15 & 12.03 & 14.10 \\
\hline Bio. & 18.04 & 11.60 & 11.06 & 10.70 & 12.85 \\
\hline Bio. + NPK 75\% & 39.04 & 27.92 & 24.01 & 20.72 & 27.92 \\
\hline Bio. + NPK 50\% & 22.16 & 17.56 & 16.74 & 13.80 & 17.57 \\
\hline Mean (A) & 26.10 & 19.04 & 17.03 & 14.66 & \\
\hline L.S.D. at $5 \%$ & \multicolumn{2}{|c|}{ A : 0.32} & B : 0.37 & \multicolumn{2}{|r|}{$\mathrm{AB}: 0.74$} \\
\hline
\end{tabular}

Bio. : Biofertilizers (Minia Azotein + phosphorein)

Table 8. Effect of cutting heights and some bio. and/or mineral NPK fertilization treatments on total dry weight (kg/plant/season) of moringa during 2015 and 2016 seasons.

\begin{tabular}{|c|c|c|c|c|c|}
\hline \multicolumn{6}{|c|}{$1^{\text {st }}$ season $(2015)$} \\
\hline \multirow{2}{*}{$\begin{array}{l}\text { Some bio. and/or mineral } \\
\text { fertilization treatments (B) }\end{array}$} & \multicolumn{5}{|c|}{ Cutting heights (cm) (A) } \\
\hline & 10 & 20 & 30 & 40 & Mean (B) \\
\hline Control & 5.27 & 2.26 & 2.15 & 1.62 & 2.83 \\
\hline NPK $100 \%$ & 12.35 & 8.37 & 7.97 & 4.97 & 8.42 \\
\hline NPK 75\% & 9.16 & 5.06 & 4.94 & 3.85 & 5.75 \\
\hline NPK $50 \%$ & 6.21 & 3.13 & 3.08 & 2.89 & 3.83 \\
\hline Bio. & 5.89 & 2.90 & 2.69 & 2.53 & 3.50 \\
\hline Bio. + NPK 75\% & 14.05 & 6.93 & 6.77 & 5.27 & 8.26 \\
\hline Bio. + NPK 50\% & 7.35 & 4.41 & 3.84 & 3.40 & 4.75 \\
\hline Mean (A) & 8.61 & 4.72 & 4.49 & 3.50 & \\
\hline L.S.D. at $5 \%$ & \multicolumn{2}{|c|}{ A : 0.23} & B : 0.85 & \multicolumn{2}{|r|}{$\mathrm{AB}: 1.70$} \\
\hline \multicolumn{6}{|c|}{$2^{\text {nd }}$ season $(2016)$} \\
\hline Control & 7.19 & 5.41 & 3.60 & 3.40 & 4.90 \\
\hline NPK $100 \%$ & 14.44 & 10.27 & 9.85 & 7.69 & 10.56 \\
\hline NPK 75\% & 9.62 & 7.27 & 6.87 & 5.59 & 7.34 \\
\hline NPK 50\% & 6.98 & 4.87 & 4.47 & 4.43 & 5.19 \\
\hline Bio. & 6.63 & 4.27 & 4.07 & 3.93 & 4.73 \\
\hline Bio. + NPK 75\% & 14.35 & 10.27 & 8.82 & 7.62 & 10.27 \\
\hline Bio. + NPK 50\% & 8.15 & 6.46 & 6.16 & 5.08 & 6.46 \\
\hline Mean (A) & 7.19 & 5.41 & 3.60 & 3.40 & 4.90 \\
\hline L.S.D. at $5 \%$ & \multicolumn{2}{|c|}{ A : 0.44} & B : 0.50 & \multicolumn{2}{|r|}{$\mathrm{AB}: 1.00$} \\
\hline
\end{tabular}

Bio. : Biofertilizers (Minia Azotein + phosphorein) 
Table 9. Effect of cutting heights and some bio. and/or mineral NPK fertilization treatments on total carbohydrates (\% d.w.) of moringa during 2015 and 2016 seasons.

\begin{tabular}{lccccc}
\hline & \multicolumn{5}{c}{$\mathbf{1}^{\text {st }}$ season (2015) } \\
\hline \multicolumn{1}{c}{$\begin{array}{c}\text { Cutting heights (cm) (A) } \\
\text { fertilization treatments (B) }\end{array}$} & $\mathbf{1 0}$ & $\mathbf{2 0}$ & $\mathbf{3 0}$ & $\mathbf{4 0}$ & Mean (B) \\
\hline Control & 14.18 & 14.10 & 13.90 & 13.85 & 14.01 \\
NPK 100\% & 21.80 & 21.74 & 20.53 & 19.49 & 20.89 \\
NPK 75\% & 18.02 & 17.94 & 17.76 & 17.69 & 17.85 \\
NPK 50\% & 17.20 & 17.13 & 16.94 & 16.88 & 17.04 \\
Bio. & 19.19 & 19.12 & 18.92 & 18.87 & 19.03 \\
Bio. + NPK 75\% & 21.59 & 21.53 & 20.32 & 19.27 & 20.68 \\
Bio. + NPK 50\% & 20.09 & 20.02 & 19.82 & 18.77 & 19.68 \\
Mean (A) & 18.87 & 18.80 & 18.31 & 17.83 & AB : 0.62 \\
L.S.D. at 5\% & A : N.S. & & B : 0.31 & & 14.03 \\
\hline & & $\mathbf{2}^{\text {nd }}$ season (2016) & & & 24.63 \\
Control & 14.17 & 14.13 & 13.94 & 13.88 & 17.92 \\
NPK 100\% & 24.81 & 24.78 & 24.51 & 24.50 & 17.09 \\
NPK 75\% & 18.08 & 18.05 & 17.86 & 17.70 & 19.10 \\
NPK 50\% & 17.23 & 17.20 & 16.66 & 16.95 & 24.52 \\
Bio. & 19.24 & 19.21 & 18.99 & 18.97 & 20.01 \\
Bio. + NPK 75\% & 24.66 & 24.63 & 24.40 & 24.39 & 19.88 \\
Bio. + NPK 50\% & 20.15 & 20.12 & 19.90 & 19.49 & AB : \\
Mean (A) & 19.76 & 19.73 & 19.51 & &
\end{tabular}

Bio. : Biofertilizers (Minia Azotein + phosphorein)

Table 10. Effect of cutting heights and some bio. and/or mineral NPK fertilization treatments on total protein (\% d.w.) of moringa during 2015 and 2016 seasons.

\begin{tabular}{lccccc}
\hline & \multicolumn{5}{c}{$\mathbf{1}^{\text {st }}$ season (2015) } \\
\hline \multicolumn{7}{c}{$\begin{array}{c}\text { Come bio. and/or mineral } \\
\text { fertilization treatments (B) }\end{array}$} & $\mathbf{1 0}$ & $\mathbf{2 0}$ & $\mathbf{3 0}$ & $\mathbf{4 0}$ & Mean (B) \\
\hline Control & 15.94 & 15.81 & 15.69 & 15.69 & 15.78 \\
NPK 100\% & 22.56 & 22.31 & 22.38 & 22.00 & 22.31 \\
NPK 75\% & 19.25 & 19.19 & 18.88 & 18.81 & 19.03 \\
NPK 50\% & 17.81 & 17.69 & 17.56 & 17.50 & 17.64 \\
Bio. & 21.13 & 20.94 & 20.81 & 20.75 & 20.91 \\
Bio. + NPK 75\% & 22.31 & 22.31 & 22.25 & 21.94 & 22.21 \\
Bio. + NPK 50\% & 21.88 & 21.69 & 21.63 & 21.50 & 21.68 \\
Mean (A) & 20.13 & 19.99 & 19.89 & 19.74 & \\
L.S.D. at 5\% & A : N.S. & & B : 0.16 & & AB : 0.32 \\
\hline & & $\mathbf{2}^{\text {nd }}$ season (2016) & & & 15.93 \\
Control & 16.13 & 16.00 & 15.88 & 15.69 & 23.10 \\
NPK 100\% & 23.31 & 23.13 & 23.13 & 22.81 & 19.72 \\
NPK 75\% & 19.88 & 19.75 & 19.69 & 19.56 & 18.33 \\
NPK 50\% & 18.50 & 18.38 & 18.25 & 18.19 & 21.64 \\
Bio. & 21.75 & 21.63 & 21.75 & 21.44 & 22.92 \\
Bio. + NPK 75\% & 23.00 & 23.00 & 23.00 & 22.69 & 22.39 \\
Bio. + NPK 50\% & 22.50 & 22.38 & 22.50 & 22.19 & 20.37 \\
Mean (A) & 20.72 & 20.61 & 20.60 & & AB : 0.38 \\
L.S.D. at 5\% & A : N.S. & & B : 0.19 & &
\end{tabular}

Bio. : Biofertilizers (Minia Azotein + phosphorein) 


\section{A.F. Ali et al.}

content it was significantly affected by the treatments of cutting heights (Table, 11). In general, cut plant at $10 \mathrm{~cm}$ was superior than other treatments. The present results in this investigation agreed with those obtained by Petroudi et al. (2011); Mendieta et al. (2013) and Ramos-Trejo et al. (2015).

Concerning the effect of mineral NPK and/or biofertilization treatments, data presented in Tables (9 to 11) indicated that all used treatments significantly increased total carbohydrates and protein \%, as well as, vit. C content comparing with control in both seasons. The best values were obtained with either mineral NPK $(100 \%)$ or bio. + $75 \%$ NPK for both total carbohydrates and protein $\%$, while, bio. + 75\% NPK followed by biofertilizers and then mineral NPK (100\%) recorded the highest values of vit. C.

The positive effect of mineral NPK on increasing total carbohydrates, protein and vit. C content were recorded by Darwish (2015) on Moringa oleifera, Mansour (2002) on Senna sulfurea, while, Zayed (2012); Darwish (2015) and Abdou et al. (2016) on Moringa oleifera, Al-Hadad et al. (2014) on Eucalyptus and Soliman et al. (2015) on Delonix regia concerning the effect of biofertilization treatments.

The interaction between main and sub plots $(A \times B)$ was significant for total carbohydrates, protein and vit. C \% in both seasons (Tables, 9 to 11). The highest values of total carbohydrates \% were obtained with cutting at 10 and $20 \mathrm{~cm}$ in combination with mineral NPK (100\%) or bio. + 75\% NPK (Table, 9). The best interaction over all were cutting at 10, 20 and $30 \mathrm{~cm}$ with NPK $(100 \%)$ or bio. $+75 \%$ NPK (Table, 10$)$. The interaction treatments of cutting at $10 \mathrm{~cm} \times$ bio. $+75 \%$ NPK or cutting at $10 \mathrm{~cm} \times$ biofertilizers or cutting at $10 \mathrm{~cm} \times$ mineral NPK (100\%) resulted the best content of vit. $\mathrm{C}$ as shown in Table (11).

Table 11. Effect of cutting heights and some bio. and/or mineral NPK fertilization treatments on vitamin C content (\% f.w.) of moringa during 2015 and 2016 seasons.

\begin{tabular}{|c|c|c|c|c|c|}
\hline \multicolumn{6}{|c|}{$1^{\text {st }}$ season $(2015)$} \\
\hline \multirow{2}{*}{$\begin{array}{l}\text { Some bio. and/or mineral } \\
\text { fertilization treatments (B) }\end{array}$} & \multicolumn{5}{|c|}{ Cutting heights (cm) (A) } \\
\hline & 10 & 20 & 30 & 40 & Mean (B) \\
\hline Control & 161 & 158 & 154 & 151 & 156 \\
\hline NPK $100 \%$ & 215 & 209 & 203 & 197 & 206 \\
\hline NPK 75\% & 201 & 196 & 191 & 185 & 193 \\
\hline NPK 50\% & 174 & 170 & 165 & 161 & 168 \\
\hline Bio. & 216 & 211 & 205 & 200 & 208 \\
\hline Bio. + NPK 75\% & 219 & 214 & 208 & 203 & 211 \\
\hline Bio. + NPK 50\% & 189 & 185 & 180 & 176 & 183 \\
\hline Mean (A) & 196 & 192 & 187 & 182 & \\
\hline L.S.D. at 5\% & & & $\mathrm{B}: 3$ & & $\mathrm{AB}: 6$ \\
\hline \multicolumn{6}{|l|}{ 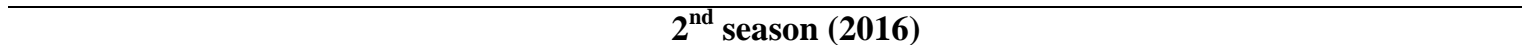 } \\
\hline Control & 165 & 162 & 158 & 153 & 160 \\
\hline NPK $100 \%$ & 218 & 214 & 205 & 201 & 209 \\
\hline NPK 75\% & 204 & 198 & 195 & 190 & 197 \\
\hline NPK 50\% & 177 & 172 & 168 & 163 & 170 \\
\hline Bio. & 222 & 217 & 211 & 205 & 214 \\
\hline Bio. + NPK 75\% & 226 & 221 & 215 & 210 & 218 \\
\hline Bio. + NPK 50\% & 192 & 187 & 183 & 179 & 185 \\
\hline Mean (A) & 201 & 196 & 191 & 186 & \\
\hline L.S.D. at 5\% & \multicolumn{2}{|c|}{$A: 3$} & B : 4 & \multicolumn{2}{|r|}{$\mathrm{AB}: 8$} \\
\hline
\end{tabular}

Bio. : Biofertilizers (Minia Azotein + phosphorein) 


\section{REFERENCES}

Abdou, M.A.H. and Ashour, R.M. (2012). Physiological studies on jojoba plants. Minia Inter. Conf. for Agric. and Irrig. In the Nile Basin countries. $26^{\text {th }}-29^{\text {th }}$ March 2012, El-Minia, Egypt.

Abdou, M.A.H.; El-Sayed, A.A.; Taha, R.A. and Mosaad, S.E.A. (2016). Response of Moringa oleifera to organic biofertilizers and mineral NPK fertilization. Scientific J. Flowers \& Ornamental Plants, 3(1):1929.

Abdullahi, I.N.; Ochi, K. and Gwaram, A.B. (2013). Plant population and fertilizer application effects on biomass productivity of Moringa oleifera in North-Central Nigeria. Peak Journal of Agricultural Sciences. 1(6):94-100.

Al-Hadad, Z.; Soliman, A.; Morsy, E.; Kamel, S. and El-Sayed, A.A. (2014). Effect of different biofertilizers and soil media on growth and chemical composition of Eucalyptus camaldulensis in North Africa. J. Hort. Sci. \& Ornamental Plants, 6(2):59-70.

Anwar, F.; Latif, S.; Ashraf, M. and Jelani, A.H. (2007). Moringa oleifera oil seed from Pakistan. J. Amr. Oil Chem. Soc., 82:45-51.

Asaolu, V.O.; Odeyinka, S.M. and Akinbamijo, O.O. (2012). The effects of four strains of mycorrhizal fungi and goat manure on fodder production by Moringa oleifera under rain-fed conditions in the Gambia-Agric. Biol. J. of North America, 3(10):391-399.

Darwish, S.N.A. (2015). Studies on Fertilizers Requirements and Planting Density for Moringa Plants in North Africa. Ph.D. Thesis, Institute of Africa Research and Studies, Department of Natural Resources, Cairo University.

Dash, S. and Gupta, N. (2009). Effect of inorganic, organic and bio-fertilizer on growth of hybrid Moringa oleifera
$\left(\mathrm{PKM}_{1}\right)$. Academic Journal of Plant Sciences 2(3):220-221.

Fagbenro, J.A.; Oshunsanya, S. O. and Onawumi, O.A. (2013). Effect of saw dust biochar and NPK 15:15:15 inorganic fertilizer on Moringa oleifera seedlings grown in an oxisol. Agrosearch, 13(1):57-68.

Foidl, N.; Mayorga, L. and Vasquez, W. (2011). Utilization del marango (Moringa oleifera) como forraje fresco para ganado. Proyecto Biomosa. Mangua. Nicaragua. Available: biomasa @ ibw.com. nihttp://www.faoorg/ag/aga/AGAP/frg/AGROFORL/Agro for.htm. Consulted: [8/12/2013].

Hedge, D.M.; Dwivedi, B.S. and Sudhakara, B.S.S. (1999). Biofertilizers for cereal production in India - A review. Indian J. Agric. Res., 69(2):73-83.

Jackson, M.L. (1973): Soil Chemical Analysis. Englewood Cliffs, New Prentice - Hall INC., New York.

Kohan, S.; Kosice, S. and Vyskumna, S. (2000). Effect of fertilizing on the production and health of poplar 1-214 (Populus $\times$ Euramericana cv. 1-214) in intensive plantations. J. For. Sci., 46(7):325-330.

Lawal, B.A.; Olawepo, T.F.; Asaolu, V.O.; Akanbi, W.B.; Jolaoso, M.A. and Ojo, A.M. (2015). Effect of different methods of etablishment on growth and biomass yield of Moringa (Moringa Oleifera, Lam). International Journal of Agriculture and Crop Sciences. 8(4): 650-653.

Mansour, H.A. (2002). Influence of soil amendments and NPK fertilization on the growth and chemical composition of Senna sulfurea plants grown in sandy soil. Annals of Agric. Sci., Moshtohor, 40(3):1779-1796.

Mendieta, A.B.E.; Sporndly, N.; ReyesSanchez; Salmeron, F. and Halling, M. 


\section{A.F. Ali et al.}

(2013). Biomass production and chemical composition of Moringa oleifera under different planting densities and levels of nitrogen fertilization. Agroforestry Systems; 87(1):81-92.

Michel, K.G.; Gilles, P.A.; Hamilton, J.K. and Smith, F. (1956). Colorimetric method for determination of sugar and related substances. Analytic Chemistry, 28(3):350.

Morton, J.F. (1991). The horseradish tree, Moringa pterygosperma (Moringaceae)A boon to arid lands, Econ. Bot, 45:318333.

Mosaad, S.E.A. (2016). Response of Moringa Plants to Some Agricultural Treatments. Ph.D. Thesis, Fac. Agric., Minia Univ., Egypt.

Moustafa, H.E.B. (2008). Influence of Some Growth Media, Biofertilization and Antioxidants on The Growth of Chorisia speciosa Tree Seedlings. Ph.D. Thesis, Fac. Agric., Minia Univ., Egypt.

MSTAT-C (1986). A microcomputer program for the design management and analysis of Agronomic Research Experiments (version 4.0), Michigan State Univ., U.S.A.

Nouman, W.M.T.; Siddiqui, S.M.; Basra, R.A.; Khan, M.E.; Oleson, H. and Munir, H. (2012). Response of Moringa oleifera to saline condition. Inter. J. of Agric. Biol., 14:757-762.

Padilla, C.; Fraga, N.; Tuero, R. and Sarduy, L. (2014). Effect of cut height on indicators of forage production of Moringa oleifera cv. Plain. Cuban Journal of Agricultural Science. 48(4):405-409.

Petroudi, E.R.; Noormohammadi, G.; Mirhadi, M.J.; Madani, H. and Mobasser, H.R. (2011). Effect of nitrogen fertilization and rice harvest height on agronomic yield indices of ratoon riceberseem clover intercropping system. AJCS, 5(5):566-574.
Ramos-Trejo, O.; Castillo-Huchín, J. and Sandoval-Gío, J.J. (2015). Effect of cutting intervals and heights on forage productivity of Moringa oleifera. Revista Bio Ciencias, 3(3):187-194.

Reyes, S.N. (2006). Moringa oleifera and Cratylia argentea : Potential fodder species for ruminantes in Nicaragua. Doctoral Thesis. Swedish University of Agricultural Sciences. Uppsala.

Roe, J.H. and Keuther, C.A. (1953). The determination of ascorbic acid in whole blood urine through 2, 4-nitrophenyl hydrazine derivative of dehydro-ascorbic acid. J. Biol. Chem., 147:399-407.

Santiesteban, R.; Tamayo, E.; Verdecia, P.; Estrada, J.; Dieguez, J.; molinet, D.; Espinosa, S.; Espinosa, A. and Cordovi, C. (2012). Influence de la altura y la frecuencia de corte en le rendimiento de Moringa oleifera. I Taller Nacional de Moringa. Instituto de Ciencia Animal, Cuba, CD-ROM Shapiro, S. and Wilk, B. 1965. An analysis of variance test for normolita (complete simples). Biometrica, 52:591.

Singh, A. (2015). Influence of NPK fertilization on growth performance of Leucaena leucocephala planted on Mine Spoil. Int. J. Environ. Bioener., 10(1):4146.

Soliman, A.Sh.; Morsy, E.M. and Massoud, O.N. (2015). Tolerance of bio-fertilized Delonix regia seedlings to irrigation intervals. J. Hort. and Forestly, 7(3):7383.

Umar, A.F. (2014). Effect of Farm-Yard Manure and Inorganic Fertilizer Application on the Coppicing Ability of Moringa oleifera (Lam.) Plantation at Gaya, Kano, Nigeria. World J. Biol. Med. Science, 1(2):37-45.

Wilde, S.A.; Corey, R.P.; Lyer, J.C. and Voigt, G.K. (1985). Soil and Plant Analysis for Tree Culture. Oxford IBH. Publishing Co. New Delhi, India. 
Zayed, M.S. (2012). Improvement of growth and nutritional quality of Moringa oleifera using different biofertilizers. Annals of Agricultural Sciences, 57(1):53-62.

$$
\begin{aligned}
& \text { تأثير إرتفاعات الحش ومعاملات التسميد المعدني و/أو الحيوي على نباتات المورنجا }
\end{aligned}
$$

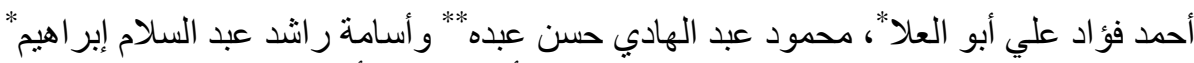

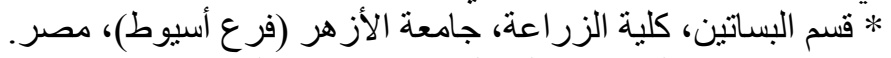

$$
\begin{aligned}
& \text { ** قسم البساتين، كلية الزر اعة، جامعة المنيا، مصر. }
\end{aligned}
$$

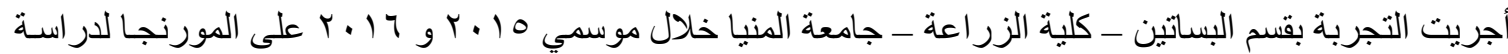

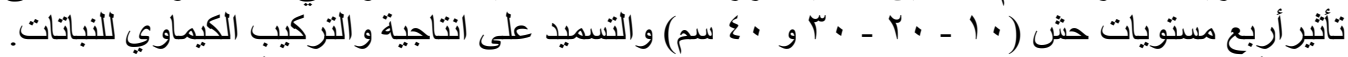

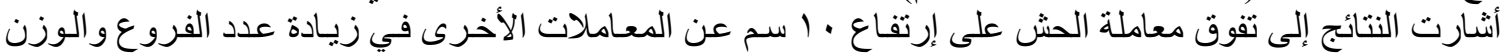

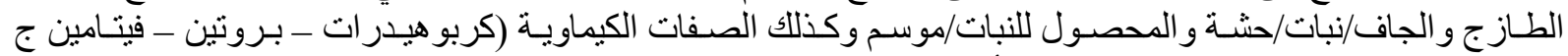

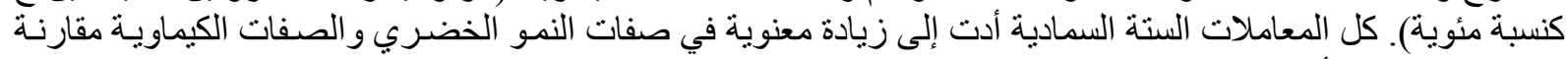

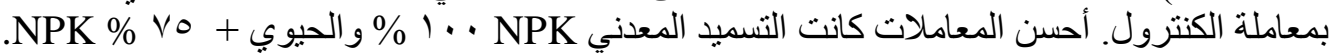

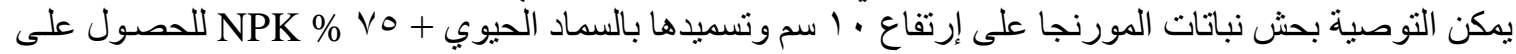
إنتاجية جيدة وبنو عية عالية. 
